\section{The nuclear receptor tailless is required for neurogenesis in the adult subventricular zone}

\author{
Hai-Kun Liu, ${ }^{1}$ Thorsten Belz, ${ }^{1}$ Dagmar Bock, ${ }^{1}$ \\ Andrea Takacs, ${ }^{1}$ Hui $\mathrm{Wu}{ }^{2}$ Peter Lichter, ${ }^{2}$ \\ Minqiang Chai, ${ }^{1}$ and Günther Schütz ${ }^{1,3}$

\begin{abstract}
${ }^{1}$ Division of Molecular Biology of the Cell I, German Cancer Research Center (DKFZ), Heidelberg 69120, Germany;

${ }^{2}$ Division of Molecular Genetics, German Cancer Research

Center (DKFZ), Heidelberg 69120, Germany
\end{abstract}

The tailless $(T 1 x)$ gene encodes an orphan nuclear receptor that is expressed by neural stem/progenitor cells in the adult brain of the subventricular zone (SVZ) and the dentate gyrus (DG). The function of Tlx in neural stem cells of the adult $S V Z$ remains largely unknown. We show here that in the SVZ of the adult brain Tlx is exclusively expressed in astrocyte-like B cells. An inducible mutation of the $T l x$ gene in the adult brain leads to complete loss of SVZ neurogenesis. Furthermore, analysis indicates that $\mathrm{Tlx}$ is required for the transition from radial glial cells to astrocyte-like neural stem cells. These findings demonstrate the crucial role of Tlx in the generation and maintenance of NSCs in the adult SVZ in vivo.

Supplemental material is available at http://www.genesdev.org.

Received March 12, 2008; revised version accepted July 9, 2008.

The subventricular zone (SVZ) of the lateral ventricle and the subgranular zone (SGZ) of the dentate gyrus (DG) are the largest germinal zones of sustained neurogenesis during adulthood in the mammalian central nervous system (Gage 2000; Alvarez-Buylla and Garcia-Verdugo 2002). Astrocyte-like type B cells in the adult SVZ are thought to be multipotent neural stem cells (NSCs) (Doetsch et al. 1999). These cells give rise to transient amplifying type $C$ cells, which in turn differentiate into type A cells (neuroblasts) that migrate to the olfactory bulb (OB) through the rostral migratory stream (RMS). The SVZOB system (Alvarez-Buylla and Garcia-Verdugo 2002) has recently been reported to also exist in the adult human brain (Curtis et al. 2007).

Tailless $(\mathrm{Tlx})$ is an orphan nuclear receptor, which is expressed in the periventricular neurogenic zone during mouse embryonic development (Monaghan et al. 1995). In the absence of this transcription factor, mutant animals survive, but suffer specific anatomical deficits in the cortex and the limbic system (Monaghan et al. 1997). Late-developing structures such as the upper cortical lay-

[Keywords: Adult neurogenesis; Tlx; self-renewal; subventricular zone] ${ }^{3}$ Corresponding author.

E-MAIL g.schuetz@dkfz.de; FAX 49-6221-42-34-70.

Article is online at http://www.genesdev.org/cgi/doi/10.1101/gad.479308. ers and the DG, are reduced in size. These phenotypic changes indicate that Tlx has an important role for brain development in the young postnatal stage. The loss of adult neurogenesis in Tlx-null mice does not exclude the possibility that this phenotype, lack of neurogenesis, is caused by the absence of Tlx during development (Shi et al. 2004). Recently, a study demonstrated that inactivation of Tlx leads to a decrease but not complete loss of neural stem cell proliferation in the DG (Zhang et al. 2008). The role of Tlx in the adult neurogenic region of the SVZ, however, remains largely unknown.

\section{Results and Discussion}

Generation of Tlx-CreER ${ }^{T 2}$ mice leads to inducible Cre activity in the $S V Z$ and $R M S$

To investigate the expression of Tlx in the adult brain, we took advantage of the BAC technology to obtain faithful expression of the tamoxifen-inducible Cre recombinase (CreER ${ }^{\mathrm{T} 2}$ fusion protein) under the control of the Tlx gene regulatory sequences. Using homologous recombination in Escherichai coli, part of exon1 of the Tlx gene was replaced by the CreER ${ }^{\mathrm{T} 2}$ cassette (Supplemental Fig. S1A). The modified BAC will express CreER ${ }^{\mathrm{T} 2}$ under the control of Tlx gene regulatory sequences contained in $104 \mathrm{~kb}$ of $5^{\prime}$ flanking sequences and $22 \mathrm{~kb}$ of $3^{\prime}$ flanking sequences. We assayed for inducible Cre recombinase translocation by tamoxifen in the $\mathrm{F} 1$ progeny from six founders. Cre recombinase is consistently found in the cytoplasm before injection and translocated into the nucleus after injection of tamoxifen (data not shown). We analyzed three lines with one, two, or three copies of the transgene (data not shown). They showed efficient Cre translocation to the nucleus after tamoxifen treatment and an identical Cre expression pattern that follows that of the endogenous Tlx gene (see below).

Tlx is expressed in the developing forebrain (Monaghan et al. 1995; Roy et al. 2004). Interestingly, it was found that the SVZ where adult neurogenesis occurs is strikingly decreased in Tlx-deficient animals (Roy et al. 2004). Since an antibody for Tlx is not available for adult brain tissue, we used an antibody against the recombinase as a reporter to follow the Tlx gene expression pattern in the adult brain via immunohistochemistry (IHC). We find activity of this reporter exclusively in sites where the Tlx gene has been found to be expressed (Monaghan et al. 1995; Shi et al. 2004). Cre is highly expressed in the SVZ (Supplemental Fig. S1B,C), as well as in the RMS (Supplemental Fig. S1B,D). We describe these cells as $\mathrm{Tlx}^{+}$.

Tlx is specifically expressed by B cells in the SVZ-OB system

NSCs and progenitors continuously produce neuroblasts, which migrate to the OB via the RMS. The cellular composition of the SVZ and RMS has been analyzed previously (Doetsch et al. 1997). The SVZ contains type B, C, and A cells and the main cell populations in the RMS are type $\mathrm{B}$ and A cells, although few $\mathrm{C}$ cells may also be found in the RMS (Doetsch et al. 2002). According to the Cre expression pattern, we found that only a subpopulation of the SVZ cells is Tlx-Cre-positive cells. To identify the 
Liu et al.

identity of these cells on the basis of Cre expression, three Tlx-CreER ${ }^{\mathrm{T} 2}$ mice were used for the following experiments; at least 10 sections were analyzed for each experiment per animal. We first analyzed sections coimmunostained for nuclear-localized Cre recombinase and SVZ cell type-specific markers. GFAP ${ }^{+}$cells in the adult SVZ have been shown to be type B cells (Doetsch et al. 1999), which has led to the concept of glial identity of NSCs. Here we show that these $\mathrm{GFAP}^{+}$cells are also $\mathrm{Cre}^{+}$, which indicates that Tlx is expressed by astrocyte-like B cells (Fig. 1A). Most EGFR ${ }^{+}$(epidermal growth factor receptor) cells in the SVZ are type C cells (Doetsch et al. 2002). Cre staining was neither detected in the EGFR ${ }^{+}$cells (Fig. 1B), nor did the staining colocalize with that of doublecortin (DCX) (Fig. 1C) or PSA-NCAM (data not shown), both of which mark A cells (Doetsch et al. 1999). It has been reported that the ependymal cells that surround the lateral ventricle may also be NSCs (Johansson et al. 1999). To determine if ependymal cells do express the Cre recombinase, we analyzed the sections with the confocal microscope. The ependymal cells that surround the lateral ventricle very tightly can be stained by DAPI (Fig. 1D), but these cells did not stain for Cre. $\mathrm{Cre}^{+}$cells are always located below the ependymal layer (Fig. 1D), establishing Cre expression in the SVZ.

We next analyzed sections coimmunostained for Cre and RMS cell type-specific markers. In the RMS, GFAP ${ }^{+}$ cells (type B cells) are $\mathrm{Cre}^{+}$(Fig. 1E). No cells costained for DCX and Cre (Fig. 1F) were detected. Since as de-
A

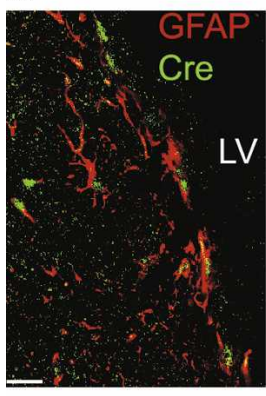

D

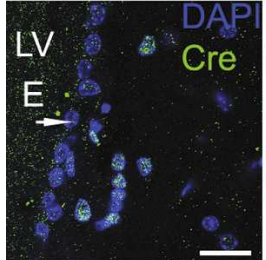

B

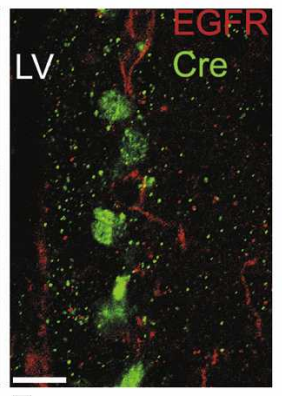

$\bar{E}$

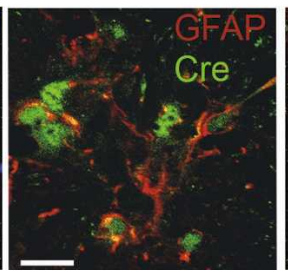

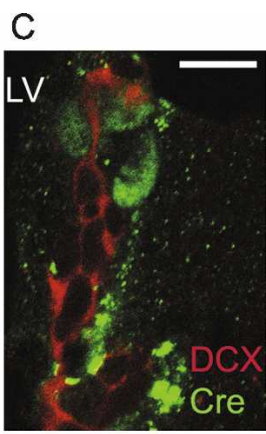

$\mathrm{F}$

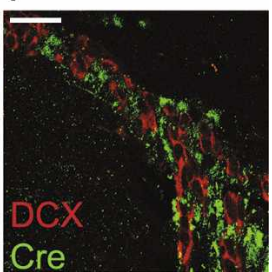

Figure 1. Tlx-CreER ${ }^{\mathrm{T} 2}$ is expressed by B cells in the adult SVZ and RMS. Tlx-CreER ${ }^{\mathrm{T} 2}$ mice were treated with tamoxifen. $(A)$ Confocal optical sections of coronal sections of the mouse SVZ coimmunostained for Cre recombinase (green) and GFAP (red). $\mathrm{Cre}^{+}$cells also express GFAP. Bar, $20 \mu \mathrm{m}$. (B) Cre recombinase (green) and EGFR (red). Cre is not costained with EGFR indicating that Tlx-CreER ${ }^{\mathrm{T} 2}$ is not expressed by $\mathrm{C}$ cells in the mouse SVZ. Bar, $10 \mu \mathrm{m}$. $(C)$ Cre (green) and DCX (red). No colabled cells were found in A cells. Bar, $10 \mu \mathrm{m} .(D)$ Cre (green) and DAPI (blue). The ependymal cells (E, arrow) do not express Cre and $\mathrm{Cre}^{+}$cells are found to reside only in the subependymal region. Bar, $10 \mu \mathrm{m} .(E)$ Confocal optical sections of coronal sections of the mouse RMS coimmunostained for Cre (green) and GFAP (red). Cre ${ }^{+}$cells are costained with GFAP as in the SVZ. Bar, $10 \mu \mathrm{m} .(F)$ Cre (green) and DCX (red). Note the absence of double-positive cells. Bar, $10 \mu \mathrm{m}$. scribed above the main cell populations of the RMS are type B and A cells, this means that Cre is only expressed by type B cells in the RMS.

To determine whether Tlx is also expressed in mature astrocytes in the cortex and OB, we also analyzed these regions. Cells colabeled for Cre and GFAP were not found, either in the cortex (data not shown), or in the OB outside the RMS region. Since Cre is only expressed in the RMS region in the OB (Supplemental Fig. S1D), we strongly conclude that $\mathrm{Tlx}$ is not a general marker for astrocytes.

\section{$\mathrm{TlX}^{+}$cells in the SVZ are multipotent}

Stem cells have the ability to self-renew and they are multipotent. To demonstrate that $\mathrm{Tlx}^{+}$cells are NSCs we marked these cells in vivo and determined whether they continuously give rise to different neural cell type. Mice carrying a floxed stop cassette before the knockin $\beta$-galactosidase $\left(\beta\right.$-gal $\left.{ }^{+}\right)$gene at the ROSA locus (Soriano 1999|, were crossed with Tlx-CreER ${ }^{\mathrm{T} 2}$ mice to allow genetic fate mapping. After tamoxifen treatment of these Tlx-CreER ${ }^{\mathrm{T}}$; R26R mice, all the $\mathrm{Tlx}^{+}$cells and their progeny will express $\beta$-galctosidase in the cytoplasm.

Tlx-CreER ${ }^{\mathrm{T} 2}$; R26R mice were injected daily with tamoxifen and $5 \mathrm{~d}$ after the last injection (in total, $10 \mathrm{~d}$ from the first injection), X-gal staining indicated efficient recombination in the SVZ and the OB where Cre is expressed (Fig. 2A). To follow the long-term fate of the marked cells, we injected tamoxifen and analyzed the animals 1 mo later. X-gal staining in the OB showed that the $\beta$-gal ${ }^{+}$cells were radially distributed in the whole OB (Fig. 2A). To identify the $\beta$-gal ${ }^{+}$cell types outside of the Cre expression region, we analyzed sections coimmunostained for $\beta$-gal and other neural cell markers. In the $\mathrm{OB}$, the vast majority of $\beta$-gal ${ }^{+}$cells are $\mathrm{NeuN}^{+}$(Fig. $2 \mathrm{~B}$ ). A few $\beta$-gal ${ }^{+}$cells were found in the corpus callosum (CC) costained with O4, an oligodendrocyte marker (Fig. $2 \mathrm{C}$. Some $\beta$-gal ${ }^{+}$cells outside of the RMS in the OB are costained with GFAP (Fig. 2D). These $\mathrm{GFAP}^{+}$cells showed more processes compared with the $\mathrm{GFAP}^{+}$in the cells of the RMS or SVZ, which are bipolar or unipolar (Garcia et al. 2004).

Recently, a report showed mosaic organization of NSCs in the SVZ of the adult brain (Merkle et al. 2007), suggesting that the NSC population in the adult SVZ is heterogeneous. To investigate if $\mathrm{Tlx}^{+}$cells include the entire B cell population or only a subpopulation, the genetic cell fate mapping approach was carried out using the Tlx-CreER ${ }^{\mathrm{T} 2}$; Z/EG mice (Novak et al. 2000), 2 mo after the last induction. We found that $\mathrm{Tlx}^{+}$cells can give rise to different kinds of neuronal cell types using neuronal markers ( $\mathrm{TH}$, calretinin, and calbindin) costained with EGFP (Supplemental Fig. S2A-C). To further investigate whether all the SVZ NSCs express $\mathrm{Tlx}$, we administered BrdU to Tlx-CreER ${ }^{\mathrm{T} 2}{ }_{\text {; }} \mathrm{R} 26 \mathrm{R}$ mice in the drinking water for 1 wk 2 mo after tamoxifen treatment. Two weeks after the BrdU treatment the mice were analyzed. If $\mathrm{Tlx}^{+}$cells will indicate all B cells within the population, all the BrdU ${ }^{+}$cells should be colabeled with $\beta$-gal. Coimmunostaing for BrdU and $\beta$-gal proved this hypothesis, since all the $\mathrm{BrdU}^{+}$cells are also $\beta$-gal ${ }^{+}$(Supplemental Fig. S2D). These results indicate that cells in the heterogeneous NSC population all express Tlx. 


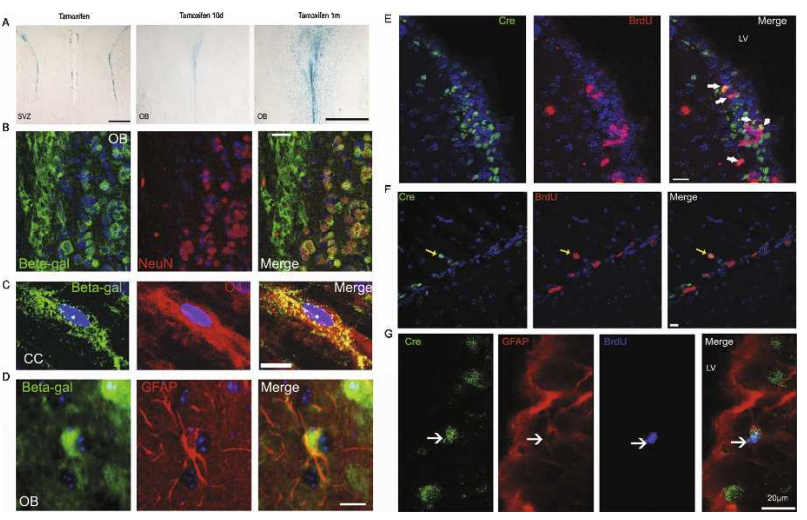

Figure 2. Adult $\mathrm{Tl} \mathrm{x}-\mathrm{Cre}^{+}$cells are multipotent and self-renewing NSCs in vivo. (A) Two-month-old mice carrying a floxed ROSA26 locus and expressing Tlx-CreER ${ }^{\mathrm{T} 2}$ were injected with tamoxifen for $5 \mathrm{~d}$, and X-gal staining of brain sections was done. Note that $5 \mathrm{~d}$ after the last tamoxifen injection, X-gal staining shows efficient recombination in the SVZ and RMS. One month after the last tamoxifen injection, $\mathrm{X}$-gal staining in OB shows radial migration of cells with the recombined locus. Bar: $B, C, 500 \mu \mathrm{m}$. $(B)$ Two-month-old TlxCreER ${ }^{\mathrm{T} 2}$;R26R mice were injected with tamoxifen for $5 \mathrm{~d}$ and 2 mo after the last tamoxifen injection, and confocal analysis of brain coronal sections were performed after coimmunostaining for $\beta$-gal ${ }^{+}$ (green) and $\mathrm{NeuN}$ (red). In $\mathrm{OB}$, most $\beta$-gal ${ }^{+}$cells are costained with NeuN outside of the RMS. Bar, $20 \mu \mathrm{m}$. $(C)$ In the corpus callosum (CC) some $\beta$-gal ${ }^{+}$cells (green) are colabeled with O4 (red), an oligodentrocyte marker. Bar, $10 \mu \mathrm{m} .(D)$ In OB, some $\beta$-gal ${ }^{+}$cells (green) outside of the RMS can be found costained with GFAP (red). Note that these cells have multipolar morphology. Bar, $10 \mu \mathrm{m}$. Confocal optical sections of 2-mo-old tamoxifen-treated Tlx-CreER ${ }^{\mathrm{T} 2}$ mice, treated with BrdU $2 \mathrm{~h}$ before sacrifice, were coimmunostained for Cre (green) and BrdU (red). (E) A low percentage (18.5\%) of $\mathrm{BrdU}^{+}$ cells is costained with Cre (arrows) in SVZ. Note that the population is simlar in size to the B-cell population. Bar, $20 \mu \mathrm{m}$. (F) Few cells double-labeled for BrdU and Cre can also be found in RMS. Bar, 10 $\mu \mathrm{m}$. $(G)$ Some BrdU LRCs express Cre and GFAP. Tlx-CreER ${ }^{\mathrm{T} 2}$ mice received BrdU continuously in the drinking water for $1 \mathrm{wk}$ followed by a 3-wk survival. Triple staining for Cre (green), GFAP (red) and BrdU (blue). Bar, $20 \mu \mathrm{m}$.

\section{$\mathrm{TlX}^{+}$cells are self-renewing stem cells in vivo}

Since Tlx is only expressed by B cells; cells costained with Cre and BrdU should be found after BrdU treatment if $\mathrm{Tlx}^{+}$cells can indeed self-renew. Tamoxifen-treated mice were injected with bromodeoxyuridine (BrdU), and sections were coimmunostained for Cre and BrdU $2 \mathrm{~h}$ after the injection. A small percentage $(18.5 \%$ when determined in five slides) of $\mathrm{BrdU}^{+}$cells are costained with Cre in SVZ (Fig. 2E), which corresponds to the B cell population as previously reported (Doetsch et al. 1997). To determine whether the B cells in the RMS are also self-renewing in vivo, we analyzed the RMS regions by coimmunostaining for Cre and BrdU. Some Tlx-Cre ${ }^{+}$B cells $3.6 \%$ when determined in 15 slides) do incorporate BrdU (Fig. 2F), which indicates that the B cells in the RMS are active NSCs in vivo, although they are less active compared with the SVZ B cells. This is consistent with previous reports demonstrating that $B$ cells are also active in the adult RMS (Gritti et al. 2002; Hack et al. 2005; Merkle et al. 2007).

Several studies reported that the slowly dividing, label-retaining cells (LRCs) correspond to the resident stem cell population (Bickenbach 1981; Morris and Potten 1994; Jackson et al. 2006). BrdU was administered in the drinking water for $1 \mathrm{wk}$ and then removed for $3 \mathrm{wk}$.
This is sufficient time for the labeled C and A cells to migrate to the OB (Jackson et al. 2006). We found that the $\mathrm{BrdU}^{+}$cells in the SVZ were also positive for GFAP and Cre (Fig. 2G); the percentage of label-retained cells among the Cre-expressing cells is $\sim 6.3 \%$ (when determined in 15 slides), which confirm that they are longterm self-renewing stem cells.

\section{Induction of Tlx ablation leads to complete loss of the self-renewal ability of NSCs in the adult SVZ}

The specificity of Tlx expression suggests that Tlx might be a crucial regulator of neurogenesis in the adult SVZ. We therefore generated mice with a conditional Tlx allele (Belz et al. 2007) that, after crossing to Tlx-CreER ${ }^{\mathrm{T} 2}$ mice, ablated the Tlx gene in the adult.

We first analyzed the targeting efficiency after tamoxifen administration. PCR analysis for the DNA isolated from the microdissected SVZ cells indicates that the recombination is efficient after induction (Supplemental Fig. S3A). The time measured after the last tamoxifen injection is expressed as weeks post-induction (WPI). To investigate the effect on cell proliferation in SVZ after induction, BrdU was injected to the mice at 4 WPI, and the proliferation frequency analyzed $2 \mathrm{~h}$ after the injection. Strikingly, cell proliferation in the SVZ is almost completely depleted in the Tlx mutant as indicated by the number of $\mathrm{BrdU}^{+}$cells (Fig. 3A,B). Further analysis of DCX-stained brain sections at 4 WPI shows a strong decrease in the number of migrating type A cells in the SVZ and in the OB of the Tlx mutant (Fig. 3C) as ex-
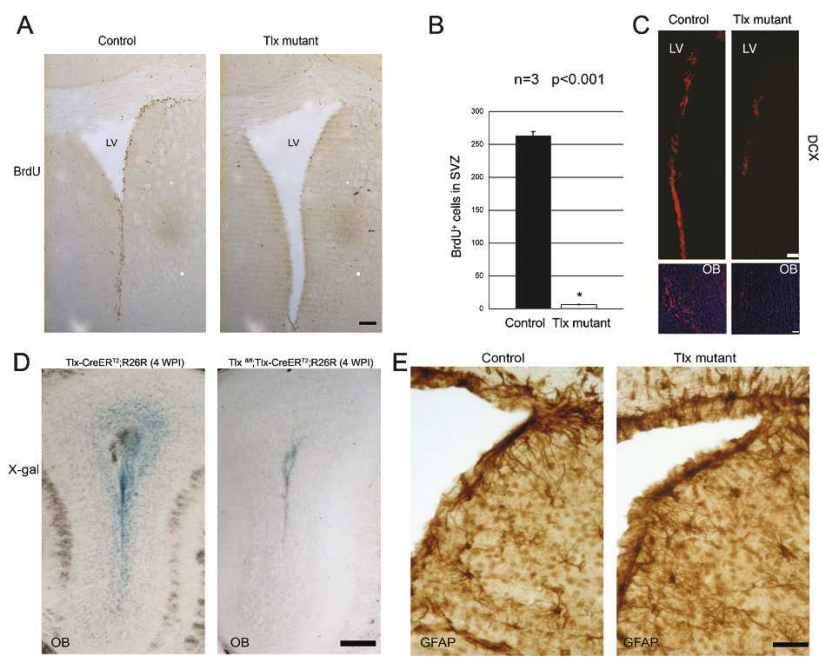

Figure 3. Tlx is essential for maintaining the self-renewal of adult NSCs in the SVZ. $(A)$ Control and Tlx mutant mice at 4 WPI were administrated with BrdU and sacrificed $2 \mathrm{~h}$ later; coronal brain sections were stained for BrdU. Note that only a few positive cells can be found in the mutant. Bar, $50 \mu \mathrm{m}$. (B) Number of BrdU+ cells in the SVZ per brain section of control and mutant at 4 WPI. $(C)$ Coronal brain sections containing the SVZ or OB were stained for DCX. Note that a dramatic decrease of DCX+cells is found in the SVZ or the OB of the Tlx mutant. Bar $10 \mu \mathrm{m}$. $(D)$ Two-month-old TlxCreERT2;R26R and Tlx-CreERT2;R26R and Tlx fl/fl mice were injected with tamoxifen for $5 \mathrm{~d}$, and after $4 \mathrm{wk}$, X-gal staining of brain sections containing the $\mathrm{OB}$ was done. Note that blue cells outside of the RMS in the Tlx fl/fl; Tlx-CreERT2;R26R mice are rarely found. Bar, $200 \mu \mathrm{m}$. (E) Coronal brain sections of control and Tlx mutant mice at 4 WPI were stained for GFAP. Note that GFAP-expressing cells are seen in the SVZ of the mutant. Bar, $20 \mu \mathrm{m}$. 
pected from the BrdU incorporation assay. To confirm the effect of loss of Tlx in SVZ by another approach independent of BrdU incorporation, we introduced the R26R reporter line in the $\mathrm{Tlx}^{f 1 / f 1}$; Tlx-CreER ${ }^{\mathrm{T} 2}$ background. As described in the genetic cell fate mapping experiment, the $\mathrm{Tlx}^{+}$cells and their progeny will carry the activated $\beta$-galactosidase gene for the entire life after tamoxifen treatment (Fig. 2A). If the loss of Tlx in the adult SVZ leads to loss of neurogenesis, we should not see any more $\beta$-galactosidase-expressing cells continuously migrating to the OB after tamoxifen induction of the Tlx ${ }^{\text {fl/f1 }}$; TlX$\mathrm{CreER}^{\mathrm{T} 2}$;R26R mice. X-gal staining at 4 WPI confirmed our hypothesis (Fig. 3D). This proves that loss of Tlx indeed causes the depletion of neurogenesis in the adult mouse SVZ. Since Tlx is exclusively expressed by type B cells, these results indicate that Tlx is crucial to maintain the self-renewal ability of adult SVZ NSCs. To investigate whether Tlx has a cell-autonomous function to NSCs, $\mathrm{Tlx}^{f 1 / f 1}$; Tlx-CreER ${ }^{\mathrm{T} 2}$;R26R mice $\left(\mathrm{Tlx}_{\mathrm{X}}-\mathrm{CreER}^{\mathrm{T} 2}\right.$; $\mathrm{R} 26 \mathrm{R}$ mice as control), $5 \mathrm{~d}$ after the last tamoxifen injection, the cells that lose Tlx expression will carry $\beta$-gal expression, and BrdU was injected to the mice to label the SVZ proliferating cells that will include the $\mathrm{C}$ and $\mathrm{A}$ cells generated before the recombination which are $\beta$ gal-negative. No significant difference was found about the number of $\mathrm{BrdU}^{+} ; \beta$-gal ${ }^{-}$cells between the control and mutant mice $2 \mathrm{~h}$ after BrdU injection (Supplemental Fig. S3B) exclude the cell-autonomous function of Tlx to the adult NSCs.

To investigate the fate of NSCs after loss of Tlx expression, we analyzed GFAP-stained brain sections at 4 WPI. There is no significant difference between the control and Tlx mutant (Fig. 3E), indicating that $\mathrm{GFAP}^{+}$cells are still found in the SVZ. We also did not observe increased cell apoptosis in the mutant, which suggests that the astrocyte-like B cells are not lost.

Loss of Tlx causes absence of NSCs in the adult brain: Tlx is required by radial glial cells to give rise to astrocyte-like B cells

Inactivation in the adult shows that $\mathrm{Tlx}$ is crucial for maintaining neurogenesis in the SVZ. We could confirm the recently published findings that loss of Tlx in the DG leads to a decrease but not loss of neurogenesis (Zhang et al. 2008). Tlx-null mice, however, lack neurogenesis in both regions. These findings suggest that the phenotype in the null mutant results from the absence of Tlx during development. Adult NSCs are derived from radial glial cells at an early postnatal stage (Anthony et al. 2004; Merkle et al. 2004; Ventura and Goldman 2007), which are the progenitors of the early developing brain.

To investigate whether the function of Tlx in radial glial cells is necessary to give rise to astrocyte-like B cells, we first analyzed brain sections from adult $\mathrm{Tlx}^{-/-}$ mice stained for GFAP and LeX/ssea1. No GFAP-expressing cells or LeX/sseal-positive cells (data not shown) are found in the SVZ-not even in the dorsal SVZ where most $\mathrm{GFAP}^{+}$are located (Fig. 4A)-but $\mathrm{GFAP}^{+}$ cells can still be found in other regions (Fig. 4A, arrows). To further investigate if the SVZ cells can still give rise to new neurons in $\mathrm{Tlx}^{-/-}$mice, BrdU was administrated to $\mathrm{Tlx}^{-/-}$mice in the drinking water for $1 \mathrm{wk}$. No BrdU ${ }^{+}$ cells were found in the brain sections of $\mathrm{Tlx}^{-/-}$mice (Fig. $4 \mathrm{~B})$, suggesting an absence of neurogenesis. Interestingly, $\mathrm{TlX}^{+/-}$mice have $38.2 \%$ less $\mathrm{BrdU}^{+}$cells than wild-type

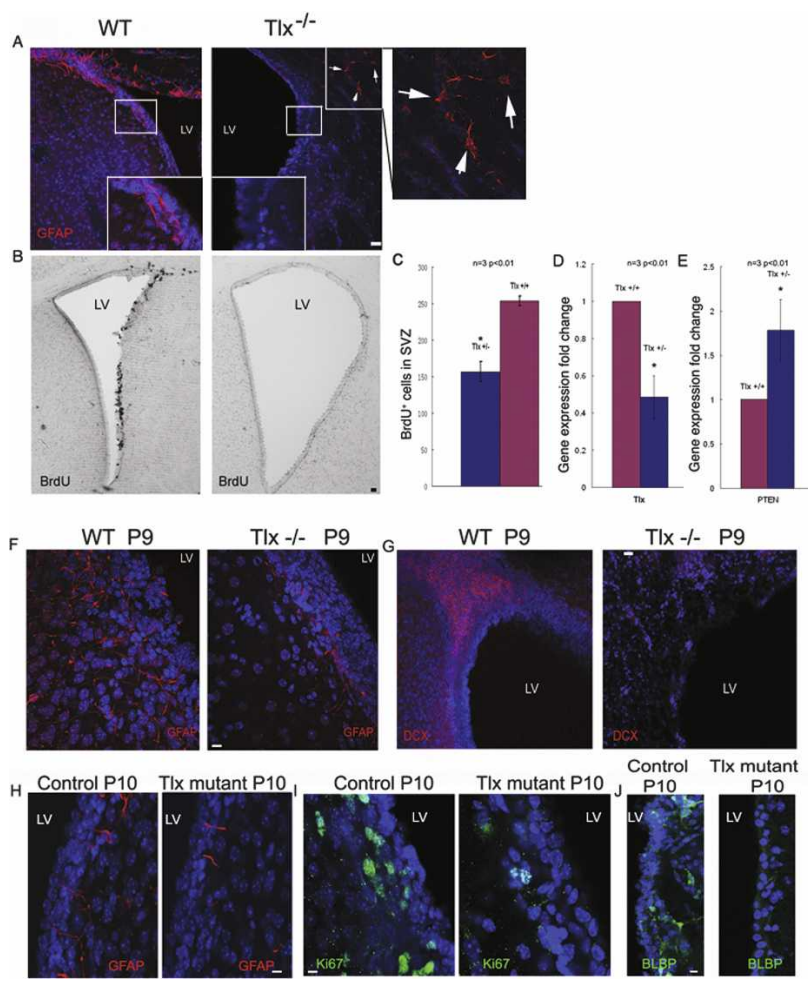

Figure 4. Tlx is required for the generation of adult NSCs. $(A)$ Coronal brain sections of 2-mo-old $\mathrm{Tlx}^{+/+}$and $\mathrm{Tlx}^{-/-}$mice were stained for GFAP (red). Note that no GFAP ${ }^{+}$cells were found in SVZ of $\mathrm{Tlx}^{-1-}$ mice, but normal astrocytes can still be found outside the SVZ (arrows). Bar, $30 \mu \mathrm{m}$. (B) BrdU. BrdU was administrated to mice for $2 \mathrm{~h}$. Note that no BrdU ${ }^{+}$cells in SVZ of $\mathrm{Tlx}^{-1-}$ mice are detected. Bar, $30 \mu \mathrm{m} .(C)$ Number of BrdU ${ }^{+}$cells in SVZ per brain section of 2-mo-old $\mathrm{Tlx}^{+/+}$and $\mathrm{Tlx}^{+/-}$mice. Note that $\mathrm{Tlx}^{+/-}$mice have $38.2 \pm 6.7 \%$ less labeled cells than $\mathrm{Tlx}^{+/+}$mice. Mean \pm standard deviation $(\mathrm{SD})$. (D) Quantitative PCR validates decreased expression of Tlx mRNA in $\mathrm{Tlx}^{+/-}(n=3)$ versus $\mathrm{Tlx}^{+/+}(n=3)$ laser-microdissected SVZ cells. Note that expression of Tlx mRNA in $\mathrm{Tlx}^{+/-}$is $48 \pm 11 \%$ of the wild type. $P<0.01$, mean \pm standard deviation $(\mathrm{SD})$. $(E)$ Quantitative PCR validates increased expression of PTEN mRNA in $\mathrm{Tlx}^{+-}(n=3)$ versus $\mathrm{Tlx}^{+/+}(n=3)$ laser microdissected SVZ cells. Note that expression of PTEN mRNA in $\mathrm{Tlx}^{+/-}$is $78 \pm 34 \%$ higher than that in $\mathrm{Tlx}^{+/+} . P<0.01$, mean \pm standard deviation $(\mathrm{SD}) .(F, G)$ Coronal brain sections of $\mathrm{P} 9 \mathrm{Tlx}^{+/+}$and $\mathrm{Tlx}^{-/-}$mice were stained for GFAP (red in $F)$. Note that almost no $\mathrm{GFAP}^{+}$cells were found in the SVZ of $\mathrm{Tlx}^{-/-}$mice. Bar, $10 \mu \mathrm{m}$. (G) DCX (red). Note that almost no $\mathrm{DCX}^{+}$cells were found in the SVZ and RMS of Tlx ${ }^{-/-}$mice. Bar, 30 um. $(H-I)$ Tamoxifen were injected to Tlx ${ }^{f l / f 1}$; Tlx-CreER ${ }^{\mathrm{T} 2}$ and $\mathrm{Tlx}^{f l / f 1}$ control mice from P5 to P8, the P10 brain sections were stained for GFAP (red in $H$ ), Ki67 (green in I), and BLBP (green in $J$ ). Note that less GFAP-, Ki67-, and BLBP-expressing cells in the mutant SVZ than the control mice. Bar, $10 \mu \mathrm{m}$.

mice in the BrdU incorporation assay, which indicates a threshold requirement for Tlx in adult neurogenesis (Fig. 4C). Phosphatase and tensin homolog (PTEN), a tumor suppressor that negatively regulates neural stem/progenitor cell proliferation by modulating G0-G1 cell cycle entry (Groszer et al. 2006), has been shown to be a target gene of Tlx (Zhang et al. 2006). We therefore analyzed the level of PTEN mRNA in wild-type and $\mathrm{Tlx}^{+/-}$ mice by real-time PCR of laser-captured microdissected cells from the SVZ. Loss of one Tlx allele leads to a proportionate increase in the level of PTEN mRNA in microdissected cells of the SVZ (Fig. 4D,E). These findings, together with chromatin immunoprecipitation 
(ChIP)-based analyses (data not shown), confirm that PTEN is a direct target gene of Tlx and explains the decrease in adult neurogenesis in the $\mathrm{Tlx}^{+/-}$mice.

We show here that there are neither $\mathrm{GFAP}^{+} \mathrm{B}$ cells nor neurogenesis in SVZ of $\mathrm{Tlx}^{-/-}$mice. Adult NSCs are derived from radial glial cells around postnatal day 9 (P9) during postnatal development (Merkle et al. 2004). RC2reactive cells and fibers appear morphologically normal in $\mathrm{Tlx}^{-/-}$mice (Roy et al. 2004), suggesting that radial glial cells are functioning normally in the absence of Tlx. This leads us to hypotheze that Tlx is involved in the transition from radial glia to astrocyte-like stem cells. To investigate if this is indeed the case, we decided to analyze $\mathrm{Tlx}^{-/-}$mice at postnatal stages. Brain sections from P9 $\mathrm{Tlx}^{-/-}$mice were stained for GFAP and DCX. GFAP-expressing cells were rarely found in the SVZ of $\mathrm{Tlx}^{-/-}$mice. In contrast, $\mathrm{GFAP}^{+}$cells already appeared at this time in the SVZ of wild-type mice (Fig. 4F). The same result was obtained for DCX staining, which indicates that P9 $\mathrm{Tlx}^{-1-}$ mice have a strong decrease in neurogenesis (Fig. 4G). To further confirm Tlx is required for the transition from radial glia to astrocyte-like B cells during the early postnatal development, the $\mathrm{Tlx}^{f l / f 1}$; Tlx-CreER ${ }^{\mathrm{T} 2}$ mice were used to inactivate Tlx specifically in postnatal brain. Tamoxifen was injected from P5 to P8 to inactivate Tlx in radial glial cells, P10 injected mice brain were analyzed by IHC. GFAP-expressing cells are strongly decreased in the mutant (Fig. $4 \mathrm{H})$, which is consistent with the results from the $\mathrm{Tlx}^{-/-}$mice. Ki67 staining indicates that loss of Tlx in radial glial cells leads to a decrease in cell proliferation (Fig. 4I). Brain lipid binding protein (BLBP) is expressed by radial glial cells in development and by astrocytes in the adult brain (Mori et al. 2005). To analyze whether the radial glial cells still exist in the mutant if they cannot transit into astrocyte-like B cells, BLBP antibody was used to stain the brain sections from the tamoxifen-injected mice. The SVZ cells in the mutant do not express BLBP like the cells from the control mice (Fig. 4J), which suggest the absence of radia glia and astrocyte-like stem cells in the SVZ of the Tlx mutant. We also tried to inactivate Tlx from P10 to P14, which means loss of Tlx after the transition from radial glia to astrocyte-like B cell, we found a similar phenotype to the adult inactivation of Tlx. The SVZ NSCs did not lose the expression of GFAP but lost the proliferation ability (data not shown). Taken all the above results together, we strongly suggest that Tlx is also required for the generation of astrocyte-like B cells in the adult brain. These results can explain why late-developing structures are also reduced in size in the Tlx-null mutant (Monaghan et al. 1997).

That GFAP ${ }^{+}$cells in the adult SVZ are multipotent NSCs is by now well accepted. However, we do not know the fundamental characteristics that distinguish neurogenic astrocytes from the vast population of nonneurogenic astrocytes elsewhere in the brain. Obviously, there is an urgent need for markers to distinguish neurogenic astrocytes from nonneurogenic astrocytes (Doetsch 2003; Merkle and Alvarez-Buylla 2006). It is also very important to conceptually distinguish stem and progenitor cells (Seaberg and van der Kooy 2003). Here, using Cre as a reporter for Tlx expression, we demonstrate that Tlx is specifically expressed by type B cells in the SVZ-OB neurogenic system. C and A cells do not express Tlx. Whether all astrocyte-like B cells in the SVZ are NSCs is still not clear, because it is difficult to study individual stem cells in vivo. Current methods to define NSCs are strongly dependent on in vitro experiments, and it has been reported that the majority of neurospheres correspond to rapidly cycling transit-amplifying cells rather than to primary and less proliferative in vivo stem cells (Doetsch et al. 2002). Therefore, the number of quiescent primary stem cells in the SVZ most likely cannot be determined by in vitro studies. The expression of Tlx in the developing and adult NSCs strongly suggests that the $\mathrm{Tlx}^{+}$cells in SVZ are indeed primary stem cells. In the SVZ of the adult brain, using an inducible knockout system, we show that Tlx is essential for selfrenewal of the SVZ NSCs. This underscores the importance of this nuclear receptor in the adult NSCs of the SVZ (see the Supplemental Material).

In summary we show in this study that the nuclear receptor Tlx is expressed with an unprecedented exclusivity in astrocyte-like B cells of the SVZ-OB system, and that it is a crucial determinant of the generation of adult NSCs in the SVZ and the SGZ. Tlx is essential for the maintenance of self-renewal of adult NSCs in the SVZ. This study also provides insights of the different identities of NSCs in the postnatal and adult brain, as well as in different neurogenic regions of the adult brain.

\section{Materials and methods}

\section{Animals}

Mice were housed according to international standard conditions, and all animal experiments conformed to local and international guidelines for the use of experimental animals.

\section{Tamoxifen, BrdU administration}

Tamoxifen (Sigma) was dissolved in sunflower seed oil (Sigma) with 10\% $\mathrm{EtOH}_{\mathrm{abs}}$ to prepare the $10 \mathrm{mg} / \mathrm{mL}$ solution. To follow Cre translocation by staining, 1-mg of tamoxifen was injected intraperitoneally and the animals were sacrificed $12 \mathrm{~h}$ later. For the analysis by X-gal staining and induction of loss of the Tlx conditional allele, tamoxifen was injected for $5 \mathrm{~d}$, twice per day. BrdU (Sigma) was dissolved in sterile $\mathrm{NaCl}$ to prepare the $15 \mathrm{mg} / \mathrm{mL}$ solution and was injected once $2 \mathrm{~h}$ before sacrifice. For provision of BrdU by feeding, BrdU was dissolved in the drinking water to prepare a $1 \mathrm{mg} / \mathrm{mL}$ solution.

The full description of materials and methods is available in the Supplemental Material.

\section{Acknowledgments}

We are grateful to Wolfgang Schmid for comments on the manuscript. We thank Monika Bock for excellent secretarial assistance. This work was supported by the "Deutsche Forschungsgemeinschaft" through CollaborativeResearch Centres SFB 488 and SFB 636, FOR Ot 165/2-2, GRK 791/1.02, and Sachbeihilfe Schu 51/7-2; by the European Union through grant LSHM-CT-2005-018652 (CRESCENDO); by the "Fonds der Chemischen Industrie"; the Bundesministerium für Bildung und Forschung (BMBF) through NGFN grants FZK 01GS01117, 01GS0477, and KGCV1/ 01GS0416; and through project number 0313074C (Systems Biology).

\section{References}

Alvarez-Buylla, A. and Garcia-Verdugo, J.M. 2002. Neurogenesis in adult subventricular zone. J. Neurosci. 22: 629-634.

Anthony, T.E., Klein, C., Fishell, G., and Heintz, N. 2004. Radial glia serve as neuronal progenitors in all regions of the central nervous system. Neuron 41: 881-890.

Belz, T., Liu, H.K., Bock, D., Takacs, A., Vogt, M., Wintermantel, T., Brandwein, C., Gass, P., Greiner, E., and Schutz, G. 2007. Inactivation of the gene for the nuclear receptor tailless in the brain preserving its function in the eye. Eur. J. Neurosci. 26: 2222-2227.

Bickenbach, J.R. 1981. Identification and behavior of label-retaining cells in oral mucosa and skin. J. Dent. Res. 60: 1611-1620. 
Curtis, M.A., Kam, M., Nannmark, U., Anderson, M.F., Axell, M.Z., Wikkelso, C., Holtas, S., van Roon-Mom, W.M., Bjork-Eriksson, T. Nordborg, C., et al. 2007. Human neuroblasts migrate to the olfactory bulb via a lateral ventricular extension. Science 315: 1243-1249.

Doetsch, F. 2003. The glial identity of neural stem cells. Nat. Neurosci. 6: $1127-1134$.

Doetsch, F., Garcia-Verdugo, J.M., and Alvarez-Buylla, A. 1997. Cellular composition and three-dimensional organization of the subventricular germinal zone in the adult mammalian brain. J. Neurosci. 17: 5046-5061.

Doetsch, F., Caille, I., Lim, D.A., Garcia-Verdugo, J.M., and AlvarezBuylla, A. 1999. Subventricular zone astrocytes are neural stem cells in the adult mammalian brain. Cell 97: 703-716.

Doetsch, F., Petreanu, L., Caille, I., Garcia-Verdugo, J.M., and AlvarezBuylla, A. 2002. EGF converts transit-amplifying neurogenic precursors in the adult brain into multipotent stem cells. Neuron 36: 10211034.

Gage, F.H. 2000. Mammalian neural stem cells. Science 287: 1433-1438.

Garcia, A.D., Doan, N.B., Imura, T., Bush, T.G., and Sofroniew, M.V. 2004. GFAP-expressing progenitors are the principal source of constitutive neurogenesis in adult mouse forebrain. Nat. Neurosci. 7: 1233-1241.

Gritti, A., Bonfanti, L., Doetsch, F., Caille, I., Alvarez-Buylla, A., Lim, D.A., Galli, R., Verdugo, J.M., Herrera, D.G., and Vescovi, A.L. 2002 Multipotent neural stem cells reside into the rostral extension and olfactory bulb of adult rodents. J. Neurosci. 22: 437-445.

Groszer, M., Erickson, R., Scripture-Adams, D.D., Dougherty, J.D., Le Belle, J., Zack, J.A., Geschwind, D.H., Liu, X., Kornblum, H.I., and $\mathrm{Wu}, \mathrm{H}$. 2006. PTEN negatively regulates neural stem cell self-renewal by modulating G0-G1 cell cycle entry. Proc. Natl. Acad. Sci. 103: $111-116$

Hack, M.A., Saghatelyan, A., de Chevigny, A., Pfeifer, A., Ashery-Padan, R., Lledo, P.M., and Gotz, M. 2005. Neuronal fate determinants of adult olfactory bulb neurogenesis. Nat. Neurosci. 8: 865-872.

Jackson, E.L., Garcia-Verdugo, J.M., Gil-Perotin, S., Roy, M., QuinonesHinojosa, A., VandenBerg, S., and Alvarez-Buylla, A. 2006. PDGFR $\alpha$-positive B cells are neural stem cells in the adult SVZ that form glioma-like growths in response to increased PDGF signaling. Neuron 51: 187-199.

Johansson, C.B., Momma, S., Clarke, D.L., Risling, M., Lendahl, U., and Frisen, J. 1999. Identification of a neural stem cell in the adult mammalian central nervous system. Cell 96: 25-34.

Merkle, F.T. and Alvarez-Buylla, A. 2006. Neural stem cells in mammalian development. Curr. Opin. Cell Biol. 18: 704-709.

Merkle, F.T., Tramontin, A.D., Garcia-Verdugo, J.M., and AlvarezBuylla, A. 2004. Radial glia give rise to adult neural stem cells in the subventricular zone. Proc. Nat1. Acad. Sci. 101: 17528-17532.

Merkle, F.T., Mirzadeh, Z., and Alvarez-Buylla, A. 2007. Mosaic organization of neural stem cells in the adult brain. Science 317: 381-384.

Monaghan, A.P., Grau, E., Bock, D., and Schutz, G. 1995. The mouse homolog of the orphan nuclear receptor tailless is expressed in the developing forebrain. Development 121: 839-853.

Monaghan, A.P., Bock, D., Gass, P., Schwager, A., Wolfer, D.P., Lipp, H.P., and Schutz, G. 1997. Defective limbic system in mice lacking the tailless gene. Nature 390: 515-517.

Mori, T., Buffo, A., and Gotz, M. 2005. The novel roles of glial cells revisited: The contribution of radial glia and astrocytes to neurogenesis. Curr. Top. Dev. Biol. 69: 67-99.

Morris, R.J. and Potten, C.S. 1994. Slowly cycling (label-retaining) epidermal cells behave like clonogenic stem cells in vitro. Cell Prolif 27: 279-289.

Novak, A., Guo, C., Yang, W., Nagy, A., and Lobe, C.G. 2000. Z/EG, a double reporter mouse line that expresses enhanced green fluorescent protein upon Cre-mediated excision. Genesis 28: 147-155.

Roy, K., Kuznicki, K., Wu, Q., Sun, Z., Bock, D., Schutz, G., Vranich, N., and Monaghan, A.P. 2004. The Tlx gene regulates the timing of neurogenesis in the cortex. J. Neurosci. 24: 8333-8345.

Seaberg, R.M. and van der Kooy, D. 2003. Stem and progenitor cells: The premature desertion of rigorous definitions. Trends Neurosci. 26: $125-131$.

Shi, Y., Chichung Lie, D., Taupin, P., Nakashima, K., Ray, J., Yu, R.T., Gage, F.H., and Evans, R.M. 2004. Expression and function of orphan nuclear receptor TLX in adult neural stem cells. Nature 427: 78-83.
Soriano, P. 1999. Generalized lacZ expression with the ROSA26 Cre reporter strain. Nat. Genet. 21: 70-71.

Ventura, R.E. and Goldman, J.E. 2007. Dorsal radial glia generate olfactory bulb interneurons in the postnatal murine brain. J. Neurosci. 27: 4297-4302.

Zhang, C.L., Zou, Y., Yu, R.T., Gage, F.H., and Evans, R.M. 2006. Nuclear receptor TLX prevents retinal dystrophy and recruits the corepressor atrophin1. Genes \& Dev. 20: 1308-1320.

Zhang, C.L., Zou, Y., He, W., Gage, F.H., and Evans, R.M. 2008. A role for adult TLX-positive neural stem cells in learning and behaviour. $\mathrm{Na}$ ture 451: 1004-1007. 


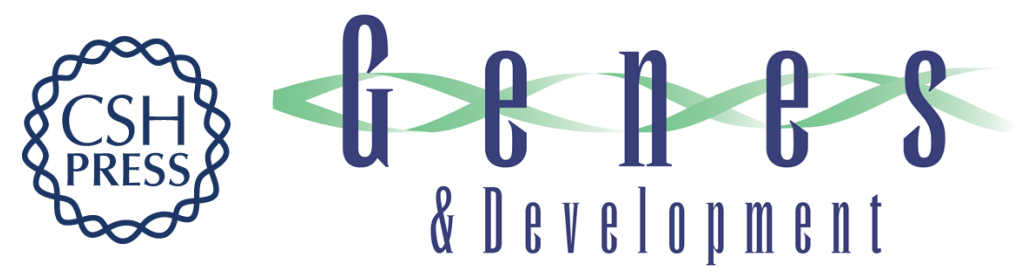

\section{The nuclear receptor tailless is required for neurogenesis in the adult subventricular zone}

Hai-Kun Liu, Thorsten Belz, Dagmar Bock, et al.

Genes Dev. 2008, 22:

Access the most recent version at doi:10.1101/gad.479308

\section{Supplemental http://genesdev.cshlp.org/content/suppl/2008/09/16/22.18.2473.DC1 Material}

References This article cites 31 articles, 12 of which can be accessed free at: http://genesdev.cshlp.org/content/22/18/2473.full.html\#ref-list-1

\section{License}

Email Alerting

Receive free email alerts when new articles cite this article - sign up in the box at the top Service right corner of the article or click here.

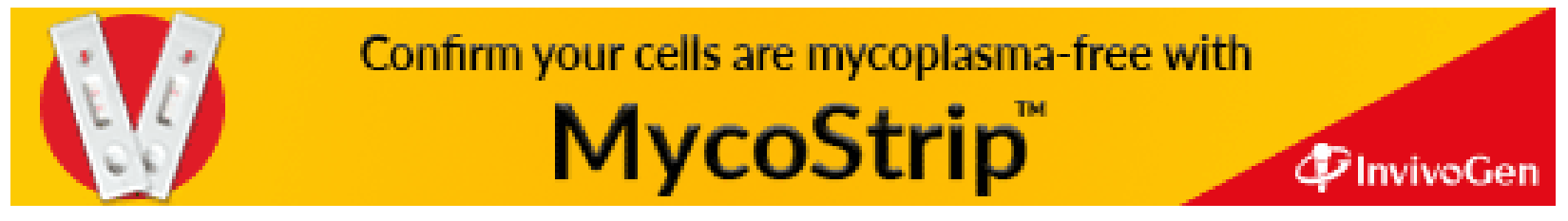

